\title{
A Low Cost Cell Calibration Technique and its PC based Control Software
}

\author{
Dr. Fernando Ribeiro \\ Industrial Electronics Dep. \\ University of Minho \\ 4800 Guimarães \\ Portugal
}

\author{
Dr. R. McMaster \\ Marine Technology \\ Cranfield University \\ Cranfield, BEDS MK43 0AL \\ United Kingdom
}

\begin{abstract}
Abstract - In this study, a technique will be presented to measure the absolute position of a robot and any other strategic positions within the workcell, and also its specially built control software.

One of the most important factors affecting the absolute or world accuracy of robots is the variation in arm geometry from a perfect kinematics form. Since arm position is essentially controlled by means of joint angles, Cartesian co-ordinates of the tool centre point are derived from the forward kinematics transform equations assuming a perfect kinematics form. Any deviation from this perfect case will result in world positional errors and hence, for effective off-line programming it is important to calibrate the real robot hardware against the virtual model used by the robot simulation computer systems.
\end{abstract}

This paper will report on work carried out in developing such low cost calibration technique, its PC Based control software and performance monitoring systems.

\section{INTRODUCTION}

To program Industrial Robots using off-line programming involves the use of a computer model of the robot and its workcell. These models need to be calibrated against the real hardware otherwise the resulting robot program won't do what it is supposed to. Although Industrial Robots have, on average, good repeatability they lack from precision in terms of absolute accuracy.

When these robots are programmed on-line, this means no problem because the user moves the robot to the correct position wherever this is. On the other hand, when using off-line programming with simulation, this becomes a critical issue since it is necessary to know exactly where the hardware of the workcell is physically, otherwise the robot programmed generated by the simulator does not correspond to the real hardware.

Therefore, there is a need to calibrate the cell and measure the absolute position of the robot as well as other locations. This paper describes details of a measuring system for recording tool point absolute positions and path trajectories.

\section{ROBOTRAK SYSTEM}

The whole system is made up of three measuring heads, an encoder interface card which is placed in a computer slot and the personal computer itself running windows.

A principle of triangulation is the basis for the ROBOTRAK system, which uses three wires, which length is always known by using an encoder, and the three measuring heads are placed at known locations within the robot envelope.

The three wires that come from the three measuring heads (one wire per measuring unit) are attached to the robot's end effector in a very precise way. The three measuring heads were in its first version attached to a precisely measured metallic plate as it can be seen in Figure 2 or Figure 3. Later on, it was found out that, that was not 
necessary if the distances between the measuring heads were precisely known. A simulation of this system can be seen in Figure 1.

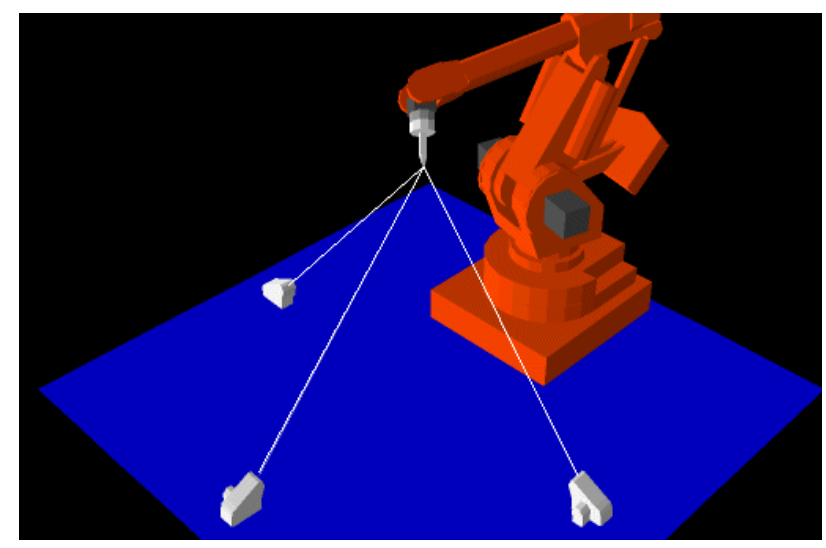

Figure 1 - Simulation of the Robotrak system with the robot and the three measuring heads

The absolute position of the robot tool point can be calculated by knowing the relative positions of the measuring heads and the three wire lengths and applying the formulas below.

$$
\begin{aligned}
& P x=\frac{\mathrm{Bx}^{2}+\mathrm{Ra}^{2}-\mathrm{Rb}^{2}}{2 \cdot \mathrm{Bx}} \\
& P y=\frac{\mathrm{Cy}^{2}+\mathrm{Ra}^{2}-\mathrm{Rc}^{2}}{2 \cdot \mathrm{Cy}} \\
& P z=\sqrt{\mathrm{Ra}^{2}-\mathrm{Px}^{2}-\mathrm{Py}^{2}}
\end{aligned}
$$

The variables used in these formulas are described in Figure 3.

By considering three spheres whose centres are located at the exit points of the hree measuring heads, one can get the correct co-ordinates by calculating the intersecting point of these three spheres. A simulation of the system with the three virtual spheres can be seen in Figure 2. The spheres were created only to help visualising the mathematical calculation.

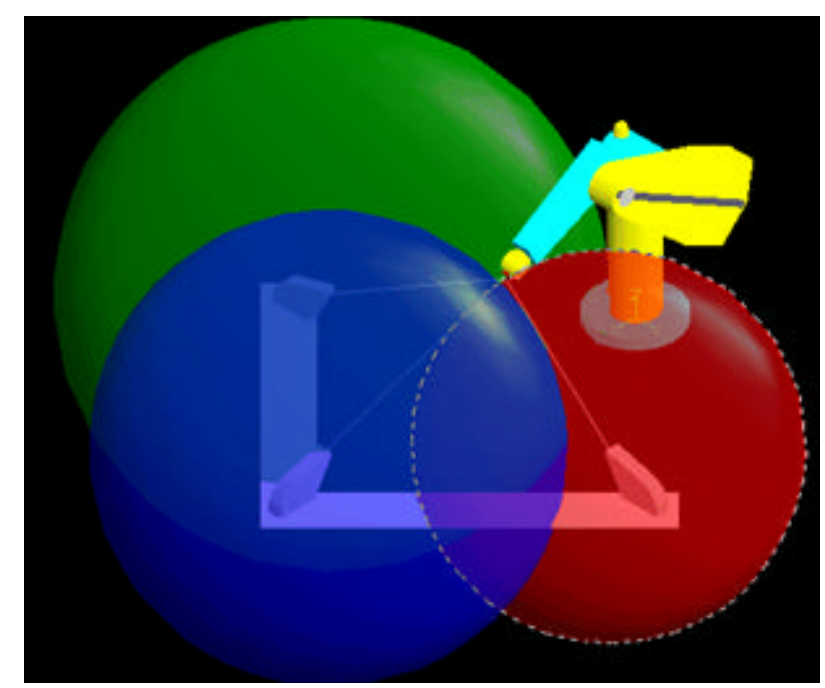

Figure 2 - Simulation of the Robotrak system with the three virtual spheres in order to help calculating the co-ordinates

An interface card is plugged onto a PC which reads the digital signals from the three encoders (measuring heads). For more information on the physical/mechanical system please refer to [1].

Then, data processing and analysis is carried out in a Personal Computer within a Windows environment and the result in provided with a graphical interface. The data can be plotted against any axis chart.

\section{ROBOTRAK SOFTWARE}

The robotrak control software here described is Windows based and very easy to use. The menu options implemented are very few in order to keep it as simple as possible. The graphical user interface is very intuitive so anybody even without any knowledge in computers can use it. The main window (with the menus) can be seen in Figure 3.

In the File menu it has the traditional options like new, open, close, save and print plus one more which is the Parameters... 


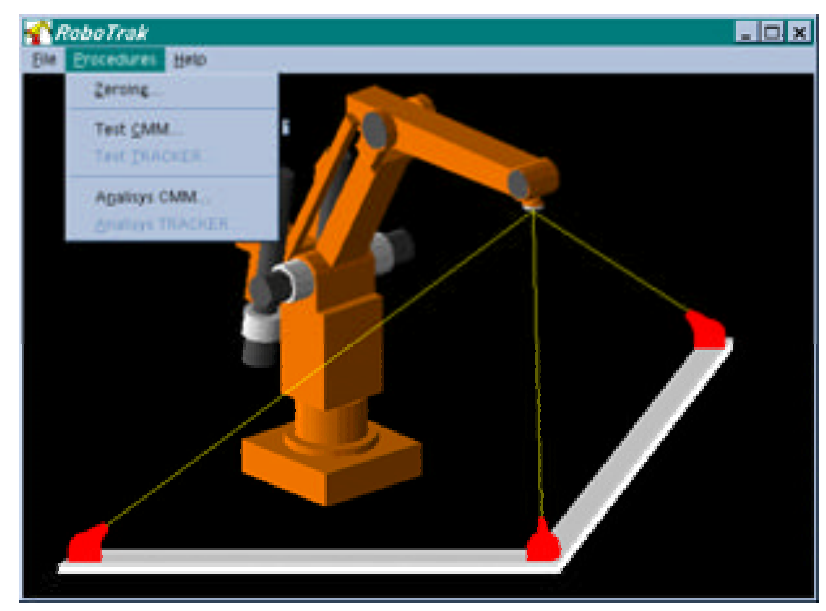

Figure 3 - Main Window of the Robotrak control Software

\section{A. Parameters Option}

This option is where the user sets up the main parameters for the desired extraction of coordinates. Figure 4 shows the parameters window.

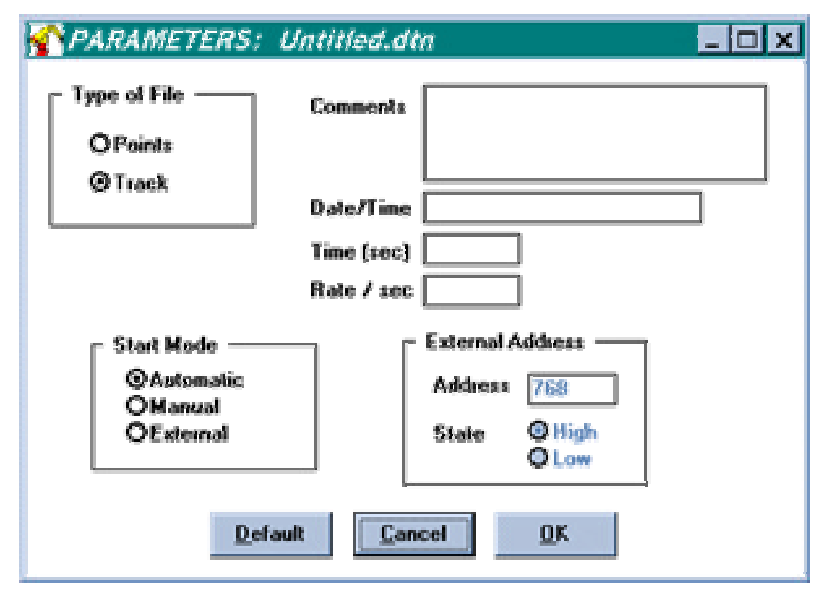

Figure 4 - Parameters Window

Here the user selects the type of extraction (points or track), comments and date/time. In the case of track being selected the user also has to select the time and rate of the extraction and also the start mode (Automatic, Manual or External). Once this is set-up the user can then go to the extraction itself.

\section{B. Zeroing Option}

The Zeroing option is used to set-up a few values of the system needed for the mathematical calculations and these are now described. This option can be seen in Figure 5.

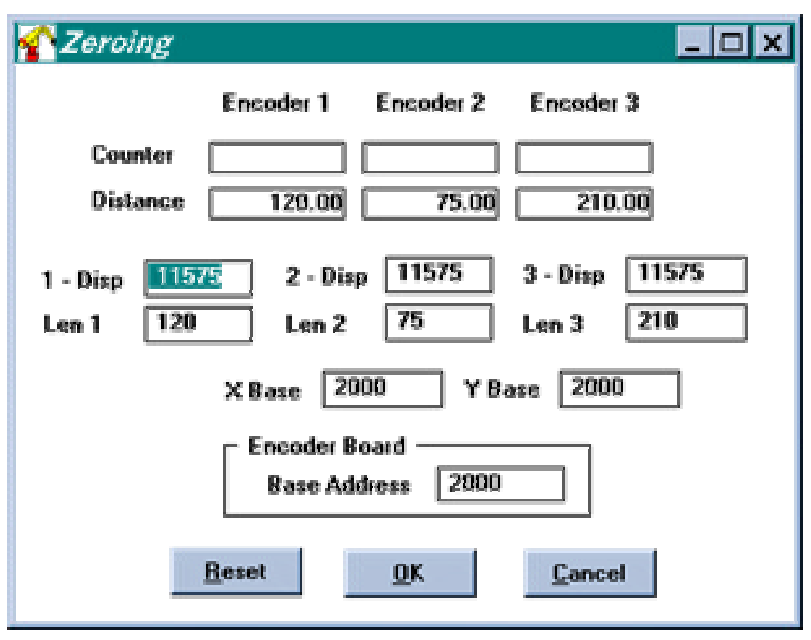

Figure 5 - Zeroing option where a few values are set-up

The distance between the measuring heads can be input in this option of the program. If the measuring heads are not attached to the metallic base the values can be updated at anytime. Also there is a small peace of wire (string) which is attached to the robot end effector to which the wire coming from the encoder is screwed to. Its length needs also to be set-up.

Also the number of pulses (counts) generated by each encoder for one meter have to be input to the program. This way, should the encoder be changed it can always be set-up to its new value.

The computer memory address of the encoders interface board can also be set up in this option.

This procedure needs only to be done once for setting up the Robotrak system and perhaps once every 6 months in order to check if those values did not change for any reason.

\section{Extraction of Point by Point Co-ordinates}

The Test CMM option is where the main procedure of the software is performed. This software works by extracting either point by point 
and recording it in a text file or by continuously reading the co-ordinates at a certain rate and stored the same way in a text file. Then, not only co-ordinates can be measured but also velocity, acceleration and tool path trajectory.

For point by point extraction, the user selects the Test $\boldsymbol{C M M}$ in the menu and a window like the one in Figure 6 appears on the screen.

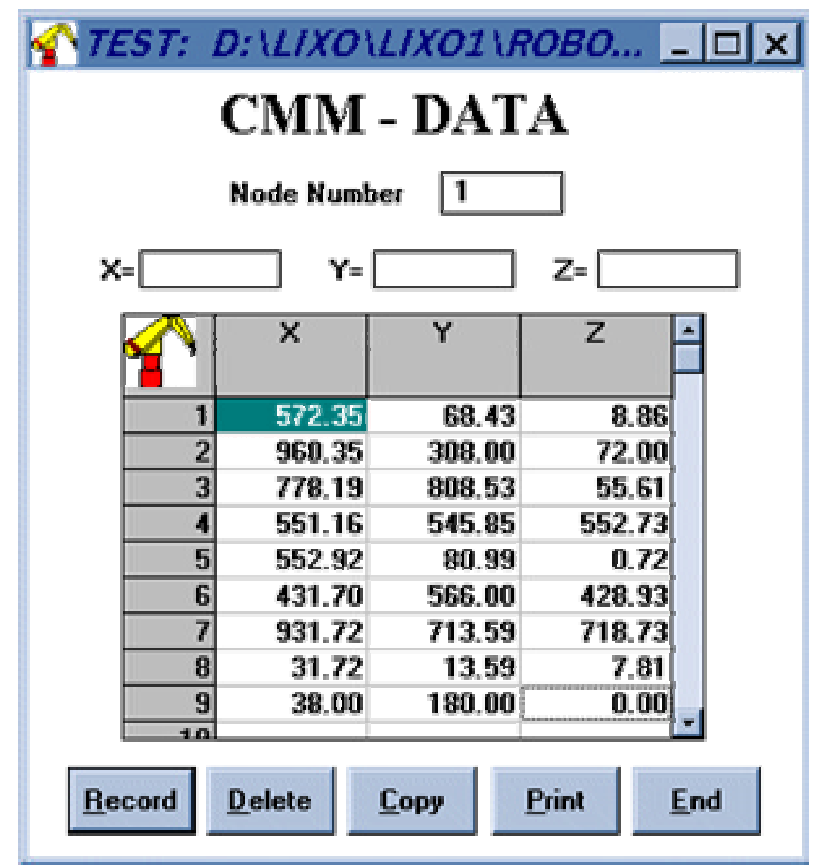

Figure 6 - Point by point extraction Robotrak software option

The user then moves the robot to the desired position and presses the Record button. The actual co-ordinates read by the encoders (and calculated by the computer) are shown on the $\mathrm{X}, \mathrm{Y}$ and $\mathrm{Z}$ fields at the top of this window. In this example these are clear because there was no robot attached to the serial port of the computer and therefore no co-ordinates were being extracted. A line is created with the co-ordinates $(X, Y, Z)$ in the spreadsheet like part of the window. Every time a user presses the Record button a new line is added. Should the user need to overwrite an existing one he just selects the node number and presses the Record button again.

There are also buttons to delete and copy a selected co-ordinate.

\section{Analysis of Point by Point co-ordinates}

The user can then select the Analysis CMM to see in a chart all the co-ordinates plotted. An example of extraction can be seen in Figure 7.

Here the co-ordinates can be accurately checked in all the axes combination possible (XY, $\mathrm{XZ}$ and $\mathrm{YZ}$ ). The scale can be changed and the charts can be printed.

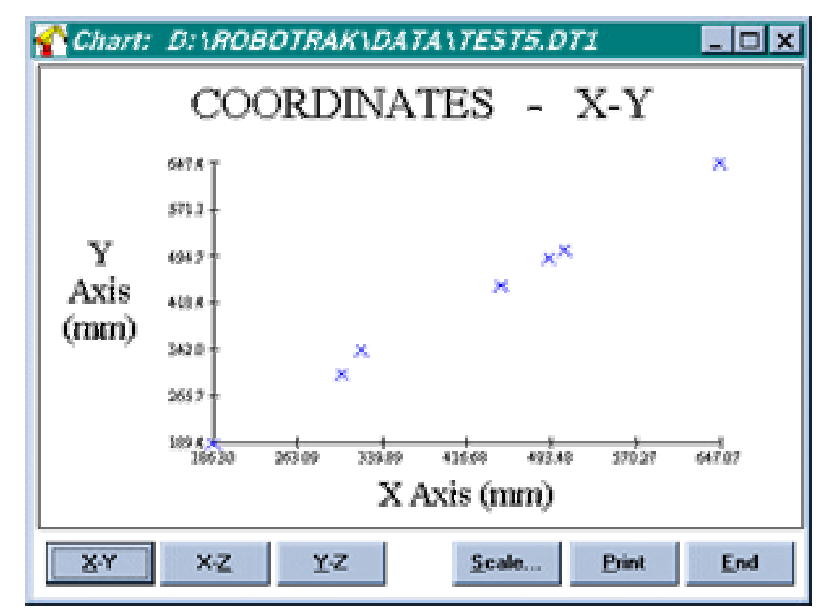

Figure 7 - Point by point analysis

\section{E. Extraction of Track}

For the track (continuous reading) extraction, the user selects the Test Track in the menu and a window like the one in Figure 6 appears on the screen.

\section{TEST: D:ULXOUXO11ROBO... $-\mid$ 回 $\mathrm{x}$} TRACKER DATA

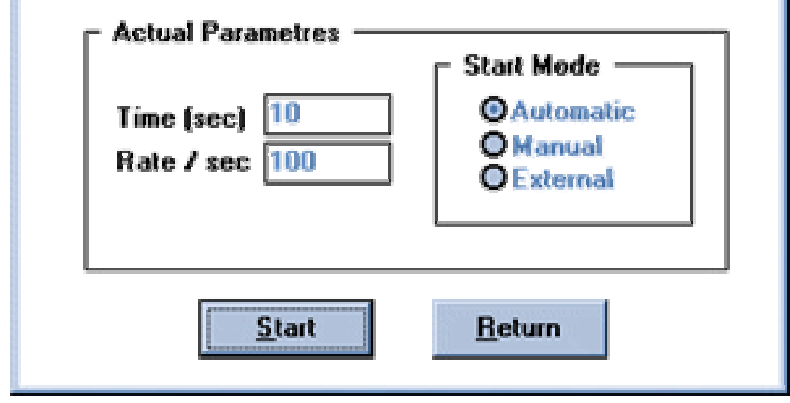

Figure 8 - Track extraction Robotrak software option 
The details for the reading are shown on the screen but cannot be changed here. They have to be changed on the parameters option.

In the Manual mode the user can press the START button and the extraction begins. If the automatic mode is selected the software starts reading as soon as the robot starts moving. In the last mode (External) another device control controls the starting (as an input to the system).

After the pre-defined time ends, the data is saved in the text file.

\section{F. Analysis of Track}

The user can analyse the data on the Track analysis menu option.

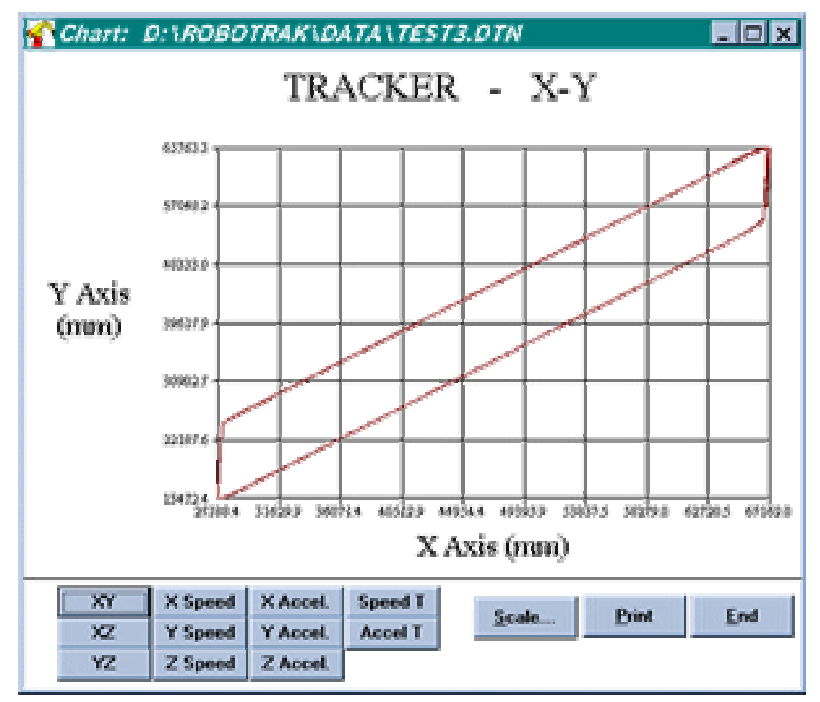

Figure 9 - Track Analysis

As it can be seen in Figure 9, the user has many charts at his choice. The actual co-ordinates plotted in all axis (XY, XY, YZ), Speed in all axes $(\mathrm{X}, \mathrm{Y}, \mathrm{Z})$, Acceleration in all axes $(\mathrm{X}, \mathrm{Y}, \mathrm{Z})$, Speed against time and Acceleration against time. The user just clicks on the desired chart and this chart is instantaneously displayed.
In any of these charts the user also has control over the scale in use and he can also print any chart.

\section{EXAMPLE OF TRACK EXTRACTION}

An track extraction sample is now described. The robot was programmed to draw a rectangle. The resulting track chart can be seen in Figure 9. The corresponding Speed on the $\mathrm{X}$ axes chart is displayed in Figure 10. It is obvious the zero speed on the $\mathrm{X}$ axis during two parts of the extraction because at that time the robot is moving in $\mathrm{X}$ axis. It then accelerates to move during its travel along the $\mathrm{Y}$ axis.

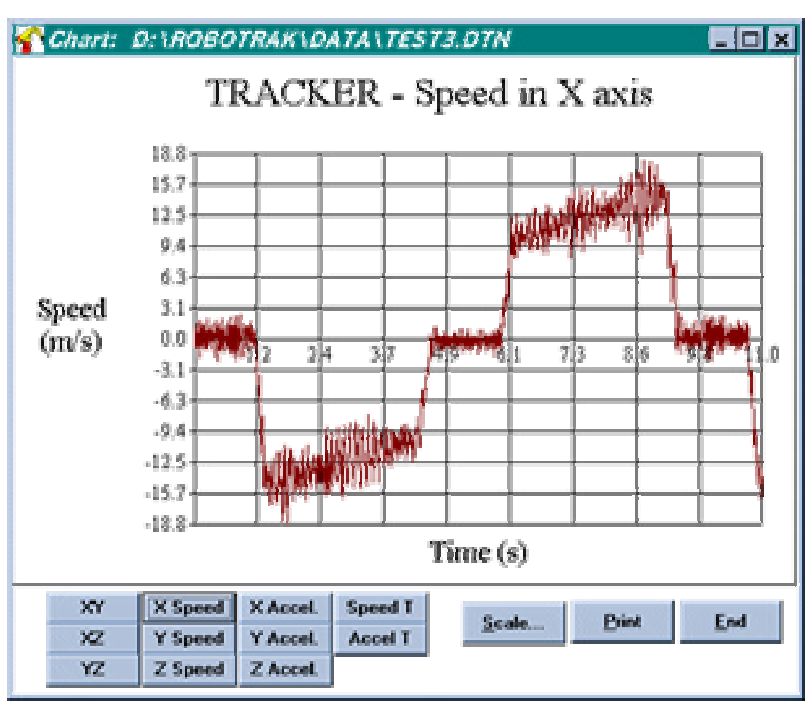

Figure 10 - Speed in $\mathrm{X}$ axis

The corresponding Speed versus time chart is displayed in Figure 11. In this chart the speed is never continuously zero since this chart is in relation to time and the robot did not stop at any time. 


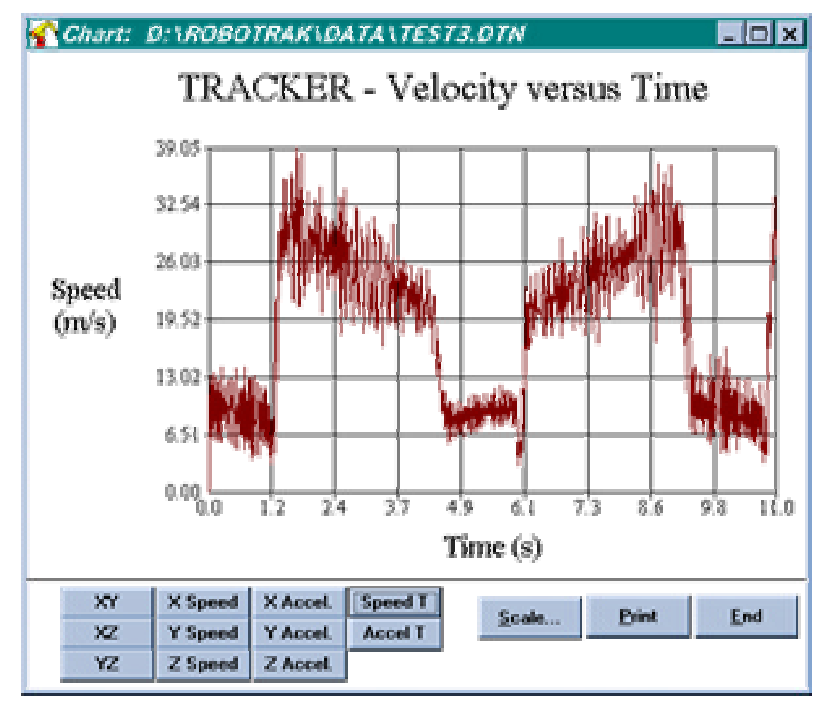

Figure 11 - Speed versus time

\section{CONCLUSIONS}

This co-ordinates extraction tool and its software proved to be very successful and easy to use as well as affordable. Several case studies were carried out and its feasibility proved to be very good. This technique is in continuous development and is now incorporated in a robot simulation package developed by Robot Simulation, Ltd at Newcastle for Personal Computers called WorkSpace which is already in its version 4.0 (for Windows 95).

\section{ACKNOWLEDGEMENTS}

First, I would like to thank my wife Marisa for encouraging and helping me on the difficult moments and my parents and sister for giving me strong support.

I would like to express my special gratitude to Dr. R. S. McMaster for his help, guidance and interest in this work. Thanks also go to the Marine Technology group for their strong support.

\section{REFERENCES}

[1] R. S. McMaster and A. Fernando Ribeiro, "Cell Calibration and Robot Tracking", IEE Colloquium 'Next Steps for Industrial Robotics', May 1994, London, England. 